\title{
SPT is Optimally Competitive for Uniprocessor Flow
}

\author{
David P. Bunde*
}

\begin{abstract}
We show that the Shortest Processing Time (SPT) algorithm is $(\Delta+1) / 2$-competitive for nonpreemptive uniprocessor total flow time with release dates, where $\Delta$ is the ratio between the longest and shortest job lengths. This is best possible for a deterministic algorithm and improves on the $(\Delta+1)$ competitive ratio shown by Epstein and van Stee using different methods.
\end{abstract}

Keywords: Algorithms; On-line algorithms; Scheduling

\section{Introduction}

We consider the problem of online nonpreemptive scheduling with release dates on a single machine to minimize total flow time $\left(1\left|r_{i}\right| \sum_{i} F_{i}\right)$. The input is a sequence of $n$ jobs, where job $J_{i}$ cannot be started before its release time $r_{i}$ and must exclusively occupy the machine for its processing time $p_{i}$. In our model, the value of $p_{i}$ is known at time $r_{i}$. Let $S_{i}^{A}$ and $C_{i}^{A}$ be the starting time and completion time of $J_{i}$ when scheduled by algorithm $A$. The flow time of job $J_{i}$ is $F_{i}^{A}=C_{i}^{A}-r_{i}$, the time between its release and completion. Our objective is to minimize $\sum_{i=1}^{n} F_{i}^{A}$, the total flow time. When preemption is allowed, i.e., jobs can be paused and resumed without penalty, the problem is solved optimally by the algorithm Shortest Remaining Processing Time (SRPT) [6, 7], which always runs the job with the least remaining processing time. When preemption is not allowed, every deterministic algorithm is $\Omega(n)$-competitive for total flow [4]. Even in the offline setting, flow cannot be approximated within a factor of $\Omega\left(n^{1 / 2-\epsilon}\right)$, for any $\epsilon>0$, unless $\mathrm{P}=\mathrm{NP}$ [4].

These strong bounds make it natural to consider approximations in terms of other parameters. One choice is $\Delta$, the ratio between the largest and smallest processing times. Epstein and van Stee [3] give bounds in terms of $\Delta$ for a resource-augmented version of the problem where an online algorithm running on $l$ processors is compared to the optimal algorithm (SRPT) running on one processor. For the special case $l=1$, they show that the algorithm Shortest Processing Time (SPT), which begins the shortest available job whenever the processor becomes idle, is $(\Delta+1)$-competitive. They also show an $\Omega(\Delta)$ lower bound for deterministic algorithms.

The main result of this paper is the following:

Theorem 1 SPT is $(\Delta+1) / 2$-competitive for total flow.

${ }^{*}$ Dept. of Computer Science, University of Illinois, Urbana-Champaign, (bunde@uiuc.edu). Partially supported by NSF grant CCR-0093348 and Sandia National Laboratories. Sandia is a multiprogram laboratory operated by Sandia Corporation, a Lockheed Martin Company, for the United States Department of Energy under Contract DE-AC04-94AL85000. 


\begin{tabular}{|c|c|c|c|}
\hline SRPT & $J_{1}\left|J_{2}\right|$ & $J_{3}$ & $J_{2}$ \\
\hline
\end{tabular}

Figure 1: SRPT schedule of 3 jobs. The active intervals of jobs $J_{1}, J_{2}$, and $J_{3}$ are $[0,2),[2,8)$, and $[3,5)$ respectively. Intervals $[0,2)$ and $[2,8)$ are maximal, so the blocks are $\left\{J_{1}\right\}$ and $\left\{J_{2}, J_{3}\right\}$.

In Section 2, we define blocks of jobs based on the SRPT schedule and show that the jobs of a block are also executed together in the SPT schedule. In Section 3 we define a block-based schedule ECHO and show it has competitive ratio $(\Delta+1) / 2$. In Section 4 we use ECHO as an intermediate between SRPT and SPT to prove Theorem 1. Then, in Section 5, we show a lower bound of $(\Delta+1) / 2$ on the competitive ratio of any deterministic algorithm. Section 6 gives concluding remarks and open problems.

\section{Block Structure}

Although typical definitions of SRPT and SPT do not specify how the algorithms choose among jobs of the same processing time, it is necessary to do so to prove our results. If SRPT has already worked on one of the jobs with equal remaining processing time, we require that it resume this job before starting the others. It may choose between jobs with equal initial processing time arbitrarily, provided that SPT uses the same order.

Now we can define blocks. The active interval of job $J_{i}$ is the half-open interval $\left[S_{i}^{S R P T}, C_{i}^{S R P T}\right)$. When two active intervals intersect, one contains the other [4]. We focus on maximal active intervals, those not contained in any other. A block is the set of jobs run during a maximal active interval. Since maximal active intervals are disjoint, the blocks partition the set of jobs. Figure 1 illustrates these definitions.

The main result of this section is that SPT obeys the block structure of SRPT; the only difference is the order in which it runs the jobs of each block. To show this, we label the blocks $B_{1}, B_{2}, \ldots, B_{m}$ in the order SRPT runs them and use $I_{i}$ to denote the interval when SRPT runs the jobs of $B_{i}$. Let the SRPT-rank of job $J$ be the index of the block containing it, i.e. $J$ has SRPT-rank $i$ if $J \in B_{i}$.

Theorem 2 SPT runs jobs in order of non-decreasing SRPT-rank.

We say that an algorithm is busy if it is idle only when it has completed all jobs that have been released. Because SRPT and SPT are both busy algorithms, they are idle at exactly the same times and Theorem 2 is equivalent to the following:

Corollary 3 For each $i$, SPT runs exactly the jobs of $B_{i}$ during interval $I_{i}$.

Proof of Theorem 2: Consider a counterexample with fewest jobs. In such a counterexample, SPT must begin a job of SRPT-rank 2 before finishing all jobs of SRPT-rank 1, because otherwise both SPT and SRPT would finish the first block of jobs at the same time and these jobs could be removed to create a smaller counterexample.

Because we specified that they use the same tie-breaking rule to select a job, SPT and SRPT both begin with the same job $J_{a}$. SRPT must preempt $J_{a}$, because otherwise this is the only job of SRPT-rank 1. Suppose SPT first starts a job of SRPT-rank 2 at time $t$ and let $J_{b}$ be the job it starts. Because we have a smallest counterexample, all jobs are started by at least one of the algorithms by time $t$. In particular, this implies that no jobs arrive after time $t$. 


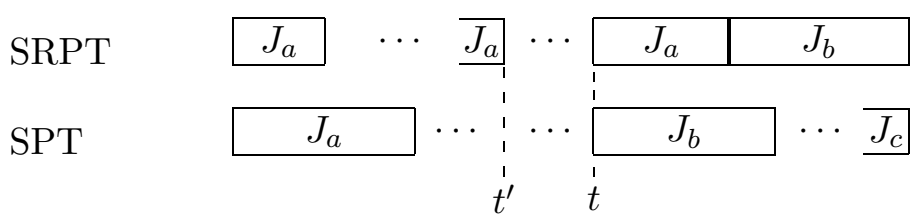

Figure 2: Illustration for the proof of Theorem 2

Let $p_{a}(t)$ be the remaining processing time of $J_{a}$ at time $t$, and let time $t^{\prime} \leq t$ be the latest time before time $t$ that SRPT works on $J_{a}$. Since SRPT works on $J_{a}$ immediately before time $t^{\prime}$, it had finished all jobs shorter than $p_{a}(t)$ available before time $t^{\prime}$. Because the jobs SRPT works on between times $t^{\prime}$ and $t$ must be shorter than $p_{a}(t)$, they arrive no earlier than time $t^{\prime}$. They are also shorter than $p_{b}$ since $J_{b} \notin B_{1}$ implies $p_{b} \geq p_{a}(t)$. Thus, SPT schedules them before $J_{b}$. Since SPT starts $J_{b}$ at time $t$, these jobs finish in time $t-t^{\prime}$, so SRPT also finishes them at time $t$ and resumes $J_{a}$. Because no jobs arrive after time $t, J_{a}$ is not interrupted. The block ends when SRPT finishes $J_{a}$ so SRPT runs $J_{b}$ next. Figure 2 depicts the situation.

SPT and SRPT are both busy so they finish the instance at the same time. SPT must run at least one job after $J_{b}$ since $p_{a}(t)>0$. Let $J_{c}$ be the last job it runs, so $J_{b}$ and $J_{c}$ finish simultaneously. Because $p_{b} \geq p_{a}(t)$, SRPT finishes $J_{a}$ no later than SPT finishes $J_{b}$ and thus starts $J_{b}$ no later than SPT starts $J_{c}$. Hence, $p_{b} \geq p_{c}$.

Recall, however, that SPT runs $J_{b}$ before $J_{c}$. $J_{c}$ was available when SPT starts $J_{b}$ at time $t$ because no jobs arrive after time $t$, implying $p_{b} \leq p_{c}$. Since SRPT runs $J_{c}$ before $J_{b}$, consistent tie breaking strengthens this to $p_{b}<p_{c}$, a contradiction.

\section{Schedule ECHO}

Now we define a new schedule ECHO in terms of the SRPT schedule. ECHO is idle at exactly the same times as SRPT. During $I_{j}$, ECHO runs the jobs of $B_{j}$, starting with the same job as SRPT and then running the others in order of increasing SRPT completion time. The jobs are run without delay so they complete during $I_{j}$. Figures 3 and 4 give examples of ECHO schedules.

For the SRPT schedule at time $t$, let first $(t)$ be the work remaining on the block's first job, $\operatorname{part}(t)$ be the work done on partially-completed jobs other than the first, and $\operatorname{curr}(t)$ be the work remaining on the currently-running job. These quantities obey the following relationship:

Lemma 4 curr $(t) \leq$ first $(t)$ - part $(t)$, with strict inequality unless SRPT is idle or working on the block's first job at time $t$.

Proof: We prove this for each block. At the start of a block, the claim holds because $\operatorname{curr}(t)=$ first $(t)$ and $\operatorname{part}(t)=0$. When SRPT does not switch jobs, the inequality remains true because $\operatorname{curr}(t)$ decreases to compensate for changes in first $(t)$ or part $(t)$. Now suppose that SRPT switches jobs at time $t$. Let $J_{i}$ be the job SRPT was running immediately before time $t$.

Case 1: $J_{i}$ is preempted to run a job $J_{j}$. Let $\operatorname{curr}^{\prime}(t)$ be the value of $\operatorname{curr}(t)$ if $J_{i}$ had not been preempted. Because $J_{j}$ preempts $J_{i}, p_{j}<\operatorname{curr}^{\prime}(t)$. If $J_{i}$ is the first job, $\operatorname{part}(t)=0$ and $\operatorname{curr}^{\prime}(t)=\operatorname{first}(t)$ imply the inequality. Otherwise, $\operatorname{curr}(t)=p_{j}<\operatorname{curr}^{\prime}(t)$ preserves it. 
Case 2: $J_{i}$ is finished and the block ends. If a new block is begun, $\operatorname{curr}(t)=f i r s t(t)$ and $\operatorname{part}(t)=0$. Otherwise, the processor is idle at time $t$, with $\operatorname{first}(t)=\operatorname{part}(t)=\operatorname{curr}(t)=0$.

Case 3: $J_{i}$ is finished, but the block does not end. Let $J_{k}$ be the unfinished job that was most recently preempted and time $t^{*}$ be when its most recent preemption occurred. By definition, SRPT finishes jobs it runs between times $t^{*}$ and $t$, so $\operatorname{part}(t)=\operatorname{part}\left(t^{*}\right)$. Also, first $(t)=$ first $\left(t^{*}\right)$ since SRPT does not run the block's first job until $J_{k}$ is finished. Because the inequality would hold at time $t^{*}$ if $J_{k}$ had not been preempted, $p_{k}\left(t^{*}\right) \leq \operatorname{first}\left(t^{*}\right)-\operatorname{part}\left(t^{*}\right)$. If $J_{k}$ is resumed at time $t, \operatorname{curr}(t)=p_{k}\left(t^{*}\right)$. Otherwise, a job shorter than $J_{j}$ runs so $\operatorname{curr}(t)<p_{k}\left(t^{*}\right)$. In either case, the inequality holds.

When SRPT is working on a job other than the block's first, the first job has been preempted and not resumed. Applying case 1 when the first job is preempted causes the inequality to become strict until the first job is resumed.

Now we are ready to prove the soundness of ECHO.

Theorem 5 ECHO does not run a job before its release time.

Proof: This is clear for the first job in each block. For other jobs, we show that ECHO only starts jobs SRPT has already finished. To see this, view the ECHO schedule as being constructed incrementally, starting with the first job in the block and adding other jobs as SRPT completes them. At any time $t$ after ECHO finishes the first job in the block, SRPT has spent first $(t)-\operatorname{part}(t)$ time on jobs that it has completed, but ECHO has not. Thus, ECHO will take time first $(t)-\operatorname{part}(t)$ to finish the already-scheduled jobs and Lemma 4 implies that SRPT finishes a job before ECHO runs out of already-scheduled jobs.

Now we consider the competitiveness of ECHO.

Lemma 6 ECHO is $(\Delta+1) / 2$-competitive for total flow.

Proof: We consider a single block starting with $J_{i}$. Let $x$ be the sum of sizes of jobs other than $J_{i}$. Let $\Sigma^{S R P T}$ and $\Sigma^{E C H O}$ denote the total flow of SRPT and ECHO, respectively. In the SRPT schedule, $J_{i}$ has flow $p_{i}+x$ and the other jobs have at least $x$, for at least $p_{i}+2 x$ total. ECHO delays $J_{i}$ by $x$ less than SRPT and delays each of the other jobs by at most $p_{i}$ more. Since there are at most $x / p_{\min }$ other jobs, where $p_{\min }$ is the instance's minimum processing time, $\Sigma^{E C H O} \leq \Sigma^{S R P T}-x+p_{i} x / p_{\min } \leq \Sigma^{S R P T}+(\Delta-1) x$. Thus, the competitive ratio is $\Sigma^{E C H O} / \Sigma^{S R P T} \leq 1+(\Delta-1) x / \Sigma^{S R P T} \leq 1+(\Delta-1) x /\left(p_{i}+2 x\right) \leq(\Delta+1) / 2$

\section{Proof of Theorem 1}

Now we prove Theorem 1 by showing that SPT generates a schedule no worse than ECHO for every problem instance. (Figure 3 gives an instance where SPT is strictly better.) Lemma 6 then implies that $\mathrm{SPT}$ is $(\Delta+1) / 2$-competitive.

To compare SPT and ECHO, consider changing a SPT schedule into an ECHO schedule by repeatedly removing the first difference between them. By Corollary 3 , it suffices to consider the schedule of a single block. Without loss of generality, assume SPT runs jobs in numerical order: $J_{1}$ followed by $J_{2}$ and so on. Let the schedules first differ at time $\tau$, when ECHO starts $J_{i}$ and SPT starts $J_{i^{\prime}}$ with $i>i^{\prime}$. We remove this difference with a slide; start $J_{i}$ at time $\tau$ and delay $J_{i^{\prime}}, \ldots, J_{i-1}$ 


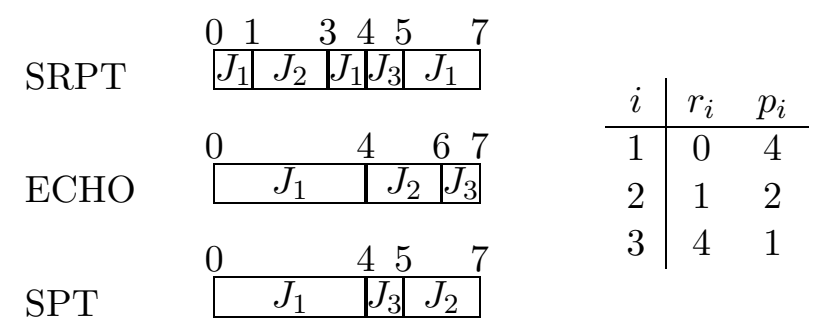

Figure 3: Instance with SPT better than ECHO $\left(\sum F_{i}^{S P T}=11\right.$ and $\left.\sum F_{i}^{E C H O}=12\right)$

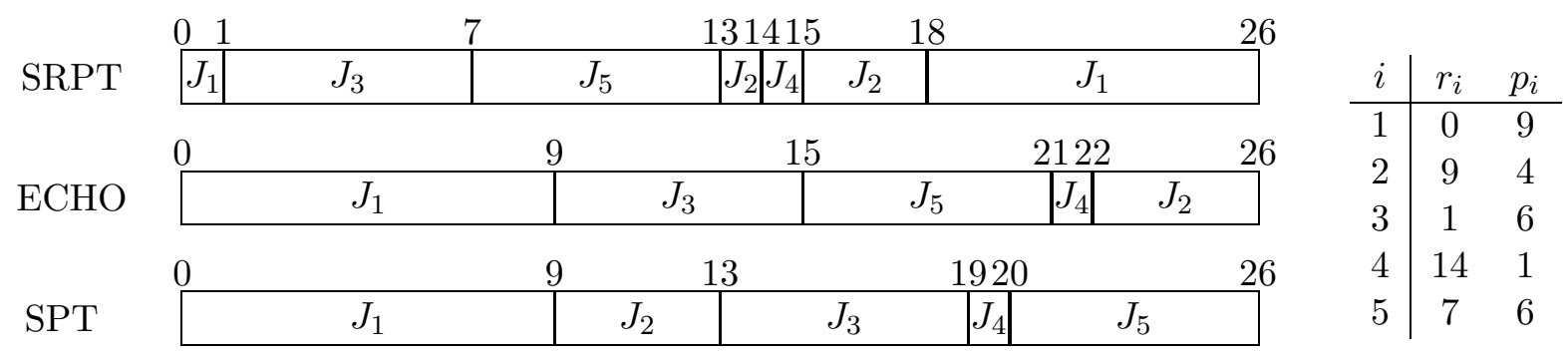

Figure 4: SRPT, ECHO, and SPT schedules

by $p_{i}$. This increases flow by $\left(i-i^{\prime}\right) p_{i}-\sum_{k=i^{\prime}}^{i-1} p_{k}=\sum_{k=i^{\prime}}^{i-1}\left(p_{i}-p_{k}\right)$. The latter expression gives the increase as the sum of increases due to each inversion, a change in relative order of $J_{i}$ and $J_{k}$. We denote an inversion moving $J_{i}$ before $J_{j}$ with $\left(J_{i}, J_{j}\right)$. For our proof, we call inversion $\left(J_{i}, J_{j}\right)$ bad if $p_{i}<p_{j}$ and good otherwise; bad inversions are those that decrease flow.

For the instance in Figure 4, changing the SPT schedule into the ECHO schedule requires 3 slide operations, advancing $J_{3}, J_{5}$, and $J_{4}$. These slides cause the inversions $\left(J_{3}, J_{2}\right),\left(J_{5}, J_{4}\right),\left(J_{5}, J_{2}\right)$, and $\left(J_{4}, J_{2}\right)$. The only bad inversion is $\left(J_{4}, J_{2}\right)$, which changes flow by $p_{4}-p_{2}=-3$.

We now show that the slide operations increase flow by proving that procedure PAIR given in Figure 5 pairs each bad inversion in which job $J_{\text {slide }}$ moves earlier with a good inversion in which $J_{\text {slide }}$ moves later so that each pair has a net increase. To demonstrate this procedure, we use it to find a partner for $\left(J_{4}, J_{2}\right)$ in the instance shown in Figure 4. $J_{\text {slide }}=J_{4}$ and only $J_{2}$ is colored blue on line 2. In the first iteration of the loop (lines 4-14), $J_{b l u e}=J_{2}, t=9, X=\left\{J_{1}\right\}$, and $Y=\left\{J_{3}\right\}$. Job $J_{3}$ is alone in $Y \backslash X$ so $J_{\text {pick }}=J_{3}$. SPT runs $J_{4}$ after $J_{3}$ so we color $J_{3}$ blue and set its note to $J_{2}$. In the second iteration, $J_{\text {blue }}=J_{3}, t=13, X=\left\{J_{1}, J_{2}\right\}$, and $Y=\left\{J_{3}, J_{5}\right\}$. The only red job in $Y \backslash X$ is $J_{5}$. SPT runs $J_{4}$ before $J_{5}$ so inversion $\left(J_{5}, J_{4}\right)$ is paired with $\left(J_{4}, \operatorname{note}\left(J_{3}\right)=J_{2}\right)$. This pair has net change in flow $\left(p_{5}-p_{4}\right)+\left(p_{4}-p_{2}\right)=2$.

We begin showing that PAIR works by proving a pair of technical lemmas.

Lemma 7 At each step, $p_{\text {blue }} \leq p_{\text {pick }}$.

Proof: $\quad$ SPT runs $J_{b l u e}$ rather than $J_{\text {pick }}$ at time $t ; J_{\text {pick }} \notin X$ so SPT has not run $J_{\text {pick }}$, but $J_{\text {pick }} \in Y$ is available because SRPT has finished it.

Lemma $8 J_{\text {slide }}$ is not released until SPT has started all the blue jobs. 
1 Procedure PAIR(job $J_{\text {slide }}$, schedule SPT, schedule SRPT)

2 color job $J$ blue if bad inversion $\left(J_{\text {slide }}, J\right)$ occurs and red otherwise

3 attach a note " $J$ " to each job $J$

4 foreach blue job $J_{b l u e}$ in SPT (in order of increasing SPT start time)

Figure 5: Procedure PAIR, which finds partners for bad inversions involving $J_{\text {slide }}$

Proof: The lemma follows from the following invariants: $(1) J_{\text {slide }}$ is shorter than each of the blue jobs, and (2) SPT runs $J_{\text {slide }}$ after all the blue jobs. Both hold initially because each blue job occurs in a bad inversion with $J_{\text {slide }}$. If $J_{\text {pick }}$ is colored blue on line 13 , the first invariant holds by Lemma 7 because the newly blue $J_{\text {pick }}$ is longer than one of the jobs that was already blue. The second invariant holds because we only color $J_{\text {pick }}$ blue if SPT runs $J_{\text {slide }}$ after it.

Now we show $J_{\text {pick }}$ can always be selected on line 8 .

Lemma 9 PAIR can always find a red job in $Y \backslash X$.

Proof: First we show there is a blue job in $X$ for each blue or green job in $Y$. Consider a job $J \in Y$ that is not red. It cannot have been colored blue on line 2 because this implies that SRPT finishes it after $J_{\text {slide }}$ and $J_{\text {slide }}$ has not been released yet by Lemma 8 . Since $J$ is not red and was not colored blue on line 2 , it was $J_{\text {pick }}$ in a previous loop iteration. The $J_{b l u e}$ from that iteration is in $X$ since line 4 considers jobs in order of SPT start time.

Now observe that $J_{1} \in X$. Since the block ends when SRPT finishes $J_{1}, J_{1} \notin Y$. Also, $J_{1}$ is never colored blue so it did not cause a $J_{\text {pick }}$ to be selected. The lemma follows since SRPT has always finished at least as many jobs as any other algorithm [6].

Next we show that the resulting pairs are valid and have non-negative flow.

Lemma 10 Each pair consists of 2 valid inversions whose combined change to flow is non-negative.

Proof: First we show that inversion $\left(J_{\text {pick }}, J_{\text {slide }}\right)$ exists. (The other inversion exists since note $\left(J_{\text {blue }}\right)$ was colored blue on line 2 .) A pair is only made if SPT runs $J_{\text {slide }}$ before $J_{\text {pick }}$. SRPT finishes $J_{\text {pick }} \in Y$ by time $t$. Since $J_{\text {slide }}$ is released after time $t$ by Lemma 8 , SRPT and ECHO run $J_{\text {slide }}$ after $J_{\text {pick }}$.

Now we show that the pair gives non-negative change in flow. Denote the processing time of the job named in note $\left(J_{i}\right)$ with $p_{\text {note }(i)}$. Then the net change of $\left\{\left(J_{\text {pick }}, J_{\text {slide }}\right),\left(J_{\text {slide }}\right.\right.$, note $\left.\left.\left(J_{\text {blue }}\right)\right)\right\}$ is $p_{\text {pick }}-p_{\text {slide }}+p_{\text {slide }}-p_{\text {note(blue })}=p_{\text {pick }}-p_{\text {note(blue })}$. By Lemma 7 , this is at least $p_{\text {blue }}-p_{\text {note(blue })}$. 
Now it suffices to show $p_{i} \geq p_{\text {note }(i)}$ for all $i$. This is initially true because note $\left(J_{i}\right)=J_{i}$. Notes are only changed on line 14 , where a note is copied from $J_{b l u e}$ to $J_{\text {pick }}$. Since $p_{\text {blue }} \leq p_{\text {pick }}$ by Lemma 7 , the claim is maintained.

Finally, we address termination.

Lemma 11 PAIR terminates and pairs all bad inversions caused by sliding $J_{\text {slide }}$.

Proof: PAIR terminates because each job is $J_{\text {blue }}$ at most once. Furthermore, since $J_{\text {pick }} \notin X$ by construction, if $J_{\text {pick }}$ is colored blue on line 13, SPT runs it after $J_{b l u e}$. Thus, each blue job creates either a pair or a later blue job. Since all blue jobs are visited, all inversions are paired.

\section{Lower Bound}

Now we show that ECHO and SPT have the best possible competitive ratio.

Theorem 12 No deterministic algorithm for nonpreemptive uniprocessor total flow is c-competitive for any fixed $c<(\Delta+1) / 2$.

Proof: For any algorithm, we construct an adversarial instance on which the algorithm's competitive ratio is arbitrarily close to $(\Delta+1) / 2$. The instance's first job has processing time $\Delta$ and release time 0. Any deterministic algorithm delays for some constant time $C$, dependent on the algorithm, and then starts this job. The instance's remaining jobs all have processing time 1 and release time $C+\epsilon+i$ for small $\epsilon$ and each $i=0, \ldots, n-2$. The algorithm has flow at least $C+\Delta+(\Delta+1-\epsilon)(n-1)$. The optimal algorithm runs the small jobs as they arrive and runs the first job either immediately if $C \geq \Delta$ or after the small jobs if $C<\Delta$. The latter case yields greater flow, $C+\Delta+2(n-1)$. The ratio between the algorithm's flow and the optimal flow approaches $(\Delta+1) / 2$ as $n \rightarrow \infty$ and $\epsilon \rightarrow 0$.

\section{Concluding Remarks}

We have shown that SPT has the best possible competitive ratio among deterministic algorithms, but it is open whether randomized algorithms can do better. The best lower bound known for randomized uniprocessor flow is $\Omega(\sqrt{\Delta})[2]$.

On a multiprocessor, SRPT does not have the same block structure because preempted jobs can be restarted on different processors. However, Awerbuch et al. [1] give an $O(\log \min \{n, \Delta\})$ competitive multiprocessor algorithm that uses preemption, but not migration. In their algorithm, each processor runs SRPT on a subset of the jobs. Replacing the SRPT schedule on each processor with ECHO removes the preemptions while increasing the competitive ratio by an $O(\Delta)$ factor. No algorithm was known to be competitive for online nonpreemptive multiprocessor flow; the best offline approximation known is $O(\sqrt{n / m} \log (n / m))$ on $m$ processors [5].

Acknowledgements The author would like to acknowledge Cynthia Phillips, Vitus Leung, Jeff Erickson, and the anonymous referees for suggestions that greatly improved this paper. 


\section{References}

[1] B. Awerbuch, Y. Azar, S. Leonardi, and O. Regev. Minimizing flow time without migration. In Proc. 31st Symp. on Theory of Computation, pages 198-205, 1999.

[2] L. Epstein and R. van Stee. Lower bounds for on-line single-machine scheduling. In Proc. 26th Symp. Math. Found. Comput. Sci., number 2136 in LNCS, pages 338-350. Springer-Verlag, 2001. http://www. informatik. uni-freiburg.de/ vanstee/papers/weight.ps.

[3] L. Epstein and R. van Stee. Optimal on-line flow time with resource augmentation. In Proc. 13th Symp. Fund. Comp. Theory, number 2138 in LNCS, pages 472-482. Springer-Verlag, 2001. http://www.math.tau.ac.il/ lea/flow.ps.gz.

[4] H. Kellerer, T. Tautenhahn, and G. J. Woeginger. Approximability and nonapproximability results for minimizing total flow time on a single machine. SIAM J. Computing, 28(4):11551166, 1999.

[5] S. Leonardi and D. Raz. Approximating total flow time on parallel machines. In Proc. 29th Symp. on Theory of Computation, pages 110-119, 1997.

[6] L. Schrage. A proof of the optimality of the shortest processing remaining time discipline. Operations Research, 16:687-690, 1968.

[7] D. Smith. A new proof of the optimality of the shortest remaining processing time discipline. Operations Research, 26(1):197-199, 1976. 